Pacific Journal of Mathematics

ON THE REPRESENTATION THEORY OF RINGS IN MATRIX 


\title{
ON THE REPRESENTATION THEORY OF RINGS IN MATRIX FORM
}

\author{
EDWARD L. GREen
}

We study the category of modules for rings of the form

$$
\left(\begin{array}{ll}
\Lambda_{1} & N \\
M & \Lambda_{2}
\end{array}\right)
$$

where $M$ is a $\Lambda_{2}-\Lambda_{1}$-bimodule and $N$ is a $\Lambda_{1}-\Lambda_{2}$-bimodule. We first obtain a structural result and then study special cases of such rings. The goal is to reduce the study of modules over such rings to modules over generalized lower triangular rings.

Rings of the form

$$
\Gamma=\left(\begin{array}{ll}
\Lambda_{1} & 0 \\
M & \Lambda_{2}
\end{array}\right)
$$

where $\Lambda_{1}, \Lambda_{2}$ are rings and $M$ is a $\Lambda_{2}-\Lambda_{1}$-bimodule have appeared often in the study of the representation theory of Artin rings and algebras. We list just a few references $([2, \S 4],[3],[4],[5, \S 9$ and appendix], [6], [7, §2], [9] and [10]). Such rings appear naturally in the study of homomorphic images of hereditary Artin algebras. For, if $\Omega$ is such an algebra, since $\Omega$ must have a simple injective left module it follows that $\Omega$ can be put in the form $(0.1)$ with $\Lambda_{1}$ a semisimple Artin ring. One of the main reasons rings of the form (0.1) are so useful is that their modules can be studied by knowing the $\Lambda_{1}$ and $\Lambda_{2}$ modules together with certain homomorphisms. In particular, we have

(0.2) THEOREM. [4] Let $\Gamma$ be the ring

$$
\left(\begin{array}{ll}
\Lambda_{1} & 0 \\
M & \Lambda_{2}
\end{array}\right)
$$

The category of left $\Gamma$-modules is equivalent to the following category: the objects are triples $(X, Y, f)$ where $X$ is a left $\Lambda_{1}$ module, $Y$ is a left $\Lambda_{2}$-module and $f \in \operatorname{Hom}_{\Lambda_{2}}\left(M \boldsymbol{\otimes}_{\Lambda_{1}} X, Y\right)$. The morphisms $\alpha:(X, Y, f) \rightarrow\left(X^{\prime}, Y^{\prime}, f^{\prime}\right)$ are pairs $\alpha=\left(\alpha_{1}, \alpha_{2}\right)$ where $\alpha_{1} \in \operatorname{Hom}_{\Lambda_{1}}\left(X, X^{\prime}\right), \alpha_{2} \in \operatorname{Hom}_{\Lambda_{2}}\left(Y, Y^{\prime}\right)$ such that the following diagram commutes 


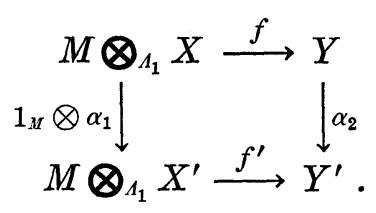

In this paper we study rings of the form

$$
\left(\begin{array}{ll}
\Lambda_{1} & N \\
M & \Lambda_{2}
\end{array}\right)
$$

where $M$ is a $\Lambda_{2}-\Lambda_{1}$-bimodule and $N$ is a $\Lambda_{1}-\Lambda_{2}$-bimodule. I. Palmer developed results on the homological and projective dimensions of such rings in [8]. The first section of this paper contains notation and a structure result which is analogous to Theorem (0.2). The remainder of the paper studies rings of form (0.3) in special cases. The goal is to reduce the study of modules over such rings to modules over generalized lower triangular rings. Under certain hypothesis this is done in the case where $\Lambda_{1}$ is a semisimple Artin ring or a left Artin ring whose Jacobson radical is square zero. A study of more general cases is conducted in $\S 3$. In $\S 4$, we give a useful application of the results of $\S 2$ and a number of examples.

1. Notation and a structure result. Throughout this paper we keep the following notation. Let $\Lambda_{1}, \Lambda_{2}$ be rings. Let $M$ be a $\Lambda_{2}-\Lambda_{1}$-bimodule and let $N$ be a $\Lambda_{1}-\Lambda_{2}$-bimodule. Let $\varphi: M \otimes_{\Lambda_{1}} N \rightarrow$ $\Lambda_{2}$ be a $\Lambda_{2}-\Lambda_{2}$-bimodule homomorphism and let $\psi: N \otimes_{\Lambda_{2}} M \rightarrow \Lambda_{1}$ be a $\Lambda_{1}-\Lambda_{1}$-bimodule homomorphism. Let

$$
\Gamma=\left(\begin{array}{ll}
\Lambda_{1} & N \\
M & \Lambda_{2}
\end{array}\right)=\left\{\left(\begin{array}{cc}
x_{1} & n \\
m & x_{2}
\end{array}\right): x_{i} \in \Lambda_{i}, m \in M, n \in N\right\} .
$$

We define addition of elements of $\Gamma$ componentwise and multiplication by

$$
\left(\begin{array}{cc}
x_{1} & n \\
m & x_{2}
\end{array}\right) \cdot\left(\begin{array}{ll}
x_{1}^{\prime} & n^{\prime} \\
m^{\prime} & x_{2}^{\prime}
\end{array}\right)=\left(\begin{array}{ll}
x_{1} x_{1}^{\prime}+\psi\left(n \otimes m^{\prime}\right) & x_{1} n^{\prime}+n x_{2}^{\prime} \\
m x_{1}^{\prime}+x_{2} m^{\prime} & x_{2} x_{2}^{\prime}+\varphi(m \otimes n)
\end{array}\right) .
$$

For $\Gamma$ to be an associative ring we must have

$$
\varphi(m \otimes n) m^{\prime}=m \psi\left(n \otimes m^{\prime}\right) \quad \text { and } \quad n \varphi\left(m \otimes n^{\prime}\right)=\psi(n \otimes m) n^{\prime}
$$

for all $m, m^{\prime} \in M$ and $n, n^{\prime} \in N$.

We henceforth assume (1.2) is always satisfied by $\varphi$ and $\psi$. With the above definitions $\Gamma$ is an (associative) ring.

If $R$ is ring, we let $\operatorname{Mod}(R)$ denote the category of left $R$ modules. We now introduce a category which we will show is 
equivalent to the category $\operatorname{Mod}(\Gamma)$. Let $\mathscr{A}(\Gamma)$ be the category whose objects are tuples $(X, Y, f, g)$ where $X \in \operatorname{Mod}\left(\Lambda_{1}\right), Y \in \operatorname{Mod}\left(\Lambda_{2}\right)$, $f \in \operatorname{Hom}_{\Lambda_{2}}\left(M \otimes_{\Lambda_{1}} X, Y\right)$ and $g \in \operatorname{Hom}_{\Lambda_{1}}\left(M \otimes_{\Lambda_{2}} Y, X\right)$ so that the following diagrams commute:
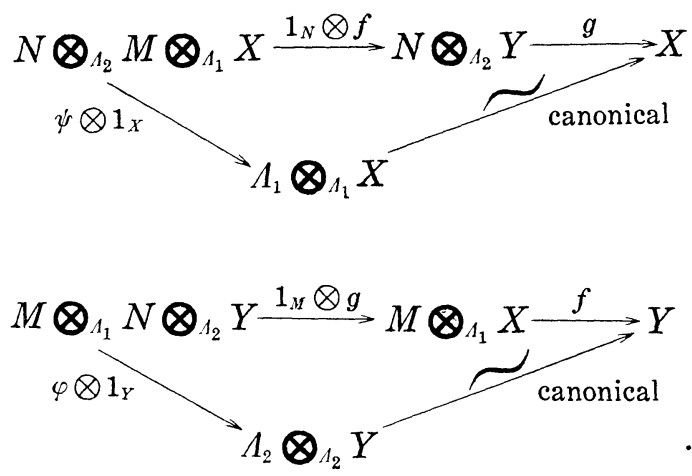

The morphisms of $\mathscr{A}(\Gamma), \alpha:(X, Y, f, g) \rightarrow\left(X^{\prime}, Y^{\prime}, f^{\prime}, g^{\prime}\right)$ are pairs of homomorphisms $\alpha=\left(\alpha_{1}, \alpha_{2}\right)$ where $\alpha_{1} \in \operatorname{Hom}_{\Lambda_{1}}\left(X, X^{\prime}\right)$ and $\alpha_{2} \in \operatorname{Hom}_{\Lambda_{2}}\left(Y, Y^{\prime}\right)$ so that the following diagrams commute:

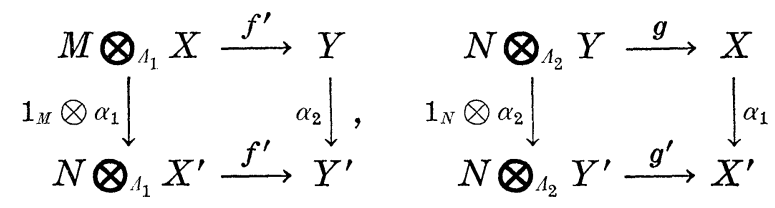

Although the proof of the following result is just a generalization of the proof of Theorem (0.2) and implicitly contained in [8], since it is of central importance to the remainder of the paper, we include a sketch of the proof for completeness.

(1.5) THEOREM. The category $\operatorname{Mod}(\Gamma)$ is equivalent to the category $\mathscr{A}(\Gamma)$.

Proof. We first define a functor $F: \operatorname{Mod}(\Gamma) \rightarrow \mathscr{A}(\Gamma)$. Let $A \in \operatorname{Mod}(\Gamma)$. Define $F(A)$ to be $\left(e_{1} A, e_{2} A, f_{A}, g_{A}\right)$ where $e_{1}=\left(\begin{array}{ll}1 & 0 \\ 0 & 0\end{array}\right), e_{2}=$ $\left(\begin{array}{ll}0 & 0 \\ 0 & 1\end{array}\right), f_{A}: M \otimes_{A_{1}} e_{1} A \rightarrow e_{2} A$ is induced from the multiplication map

$$
\left(\begin{array}{ll}
0 & 0 \\
M & 0
\end{array}\right) \bigotimes_{\Gamma} A \longrightarrow A
$$

and $g_{A}: N \otimes_{\Lambda_{2}} e_{2} A \longrightarrow e_{1} A$ is induced from the multiplication map

$$
\left(\begin{array}{ll}
0 & N \\
0 & 0
\end{array}\right) \otimes_{\Gamma} A \longrightarrow A
$$


Note that $f_{A}$ and $g_{A}$ satisfy (1.3) by (1.2). If $A, B \in \operatorname{Mod}(\Gamma)$ and $\delta \in \operatorname{Hom}_{\Gamma}(A, B)$ define $F(\delta)$ to be $\left(\delta_{1}, \delta_{2}\right)$ where $\delta_{i}$ is induced from $\delta$ noting that $\delta\left(e_{i} A\right)=e_{i} \delta\left(e_{i} A\right) \subseteq e_{i} B$. Condition (1.4) is satisfied by $F(\delta)$ since $\delta$ is a $\Gamma$-homomorphism.

We now define $G: \mathscr{A}(\Gamma) \rightarrow \operatorname{Mod}(\Gamma)$ as follows. Let $(X, Y, f, g) \in$ $\mathscr{A}(\Gamma)$. Define $G(X, Y, f, g)$ to be $\left\{\left(\begin{array}{l}x \\ y\end{array}\right): x \in X, y \in Y\right\}$. Let $\Gamma$ act on $G(X, Y, f, g)$ by

$$
\left(\begin{array}{cc}
\lambda_{1} & n \\
m & \lambda_{2}
\end{array}\right)\left(\begin{array}{l}
x \\
y
\end{array}\right)=\left(\begin{array}{l}
\lambda_{1} x+g(n \otimes y) \\
f(m \otimes x)+\lambda_{2} y
\end{array}\right)
$$

The reader may easily verify that $G(X, Y, f, g)$ is in fact a left $\Gamma$ module. If $\left(\alpha_{1}, \alpha_{2}\right):(X, Y, f, g) \rightarrow\left(X^{\prime}, Y^{\prime}, f^{\prime}, g^{\prime}\right)$ is an $\mathscr{A}(\Gamma)$-morphism we define $G\left(\alpha_{1}, \alpha_{2}\right)$ by $G\left(\alpha_{1}, \alpha_{2}\right)\left(\begin{array}{l}x \\ y\end{array}\right)=\left(\begin{array}{l}\alpha_{1}(x) \\ \alpha_{2}(y)\end{array}\right)$ for $x \in X, y \in Y$. Again it is easy to verify that $G\left(\alpha_{1}, \alpha_{2}\right)$ is a $\Gamma$-homomorphism.

Finally we leave it to the reader to check that $G F$ is naturally equivalent to $1_{\text {Mod }(\Gamma)}$ and $F G$ is naturally equivalent $1_{A(\Gamma)}$.

Note that if either $M=0$ or $N=0$ then (1.2) and (1.3) are vacuously satisfied. Thus, in either of these cases, Theorem (1.5) reduces to Theorem (0.2). We will need another structure result. Consider the generalized lower triangular ring

$$
\Sigma=\left(\begin{array}{ccc}
R_{1} & 0 & 0 \\
U & R_{2} & 0 \\
W & V & R_{3}
\end{array}\right)
$$

where each $R_{i}$ is a ring, $U$ is an $R_{2}-R_{1}$-bimodule, $V$ is an $R_{3}-R_{2^{-}}$ bimodule and $W$ is an $R_{3}-R_{1}$-bimodule. Addition in $\Sigma$ is defined componentwise and multiplication is defined by

$$
\left(\begin{array}{lll}
r_{1} & 0 & 0 \\
u & r_{2} & 0 \\
w & v & r_{3}
\end{array}\right)\left(\begin{array}{lll}
r_{1}^{\prime} & 0 & 0 \\
u^{\prime} r & 2 & 0 \\
w^{\prime} & v^{\prime} & r_{3}^{\prime}
\end{array}\right)=\left(\begin{array}{lll}
r_{1} r_{1}^{\prime} & 0 & 0 \\
u r_{1}^{\prime}+r_{2} u^{\prime} & r_{2} r_{2}^{\prime} & 0 \\
w r_{1}^{\prime}+\bar{\psi}\left(v \otimes u^{\prime}\right)+r_{3} w^{\prime} & v r_{2}^{\prime}+r_{3} v^{\prime} & r_{3} r_{3}^{\prime}
\end{array}\right)
$$

where $\bar{\psi}: V \otimes_{R_{2}} U \rightarrow W$ is an $R_{3}-R_{1}$-bimodule homomorphism.

If $\Sigma$ is of form (1.6) we define $\mathscr{B}(\Sigma)$ to be the category whose objects are tuples $(X, Y, Z, f, g, h)$ where $X \in \operatorname{Mod}\left(R_{1}\right), Y \in \operatorname{Mod}\left(R_{2}\right)$, $Z \in \operatorname{Mod}\left(R_{3}\right), f \in \operatorname{Hom}_{R_{2}}\left(U \otimes_{R_{1}} X, Y\right), g \in \operatorname{Hom}_{R_{3}}\left(V \otimes_{R_{2}} Y, Z\right)$ and $h \in$ $\mathrm{Hom}_{R_{3}}\left(W \otimes_{R_{1}} X, Z\right)$ so that the following diagram commutes

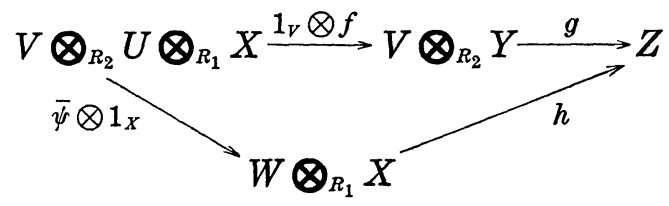


The morphisms of $\mathscr{B}(\Sigma)$ are triples $\left(\alpha_{1}, \alpha_{2}, \alpha_{3}\right):(X, Y, Z, f, g, h) \rightarrow$ $\left(X^{\prime}, Y^{\prime}, Z^{\prime}, f^{\prime}, g^{\prime}, h^{\prime}\right)$ where $\alpha_{1} \in \operatorname{Hom}_{R_{1}}\left(X, X^{\prime}\right), \alpha_{2} \in \operatorname{Hom}_{R_{9}}\left(Y, Y^{\prime}\right)$ and $\alpha_{3} \in \operatorname{Hom}_{R_{3}}\left(Z, Z^{\prime}\right)$ so that the following diagrams commute:

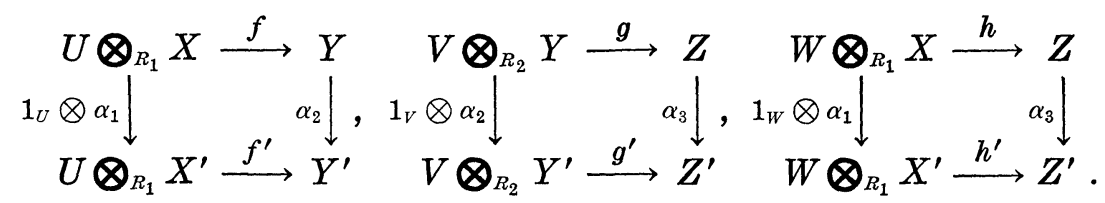

We get

(1.10) THEOREM. Let $\Sigma$ be a ring of the form (1.6). The categories $\operatorname{Mod}(\Sigma)$ and $\mathscr{B}(\Sigma)$ are equivalent.

Proof. The reader may either apply Theorem (0.2) twice, to

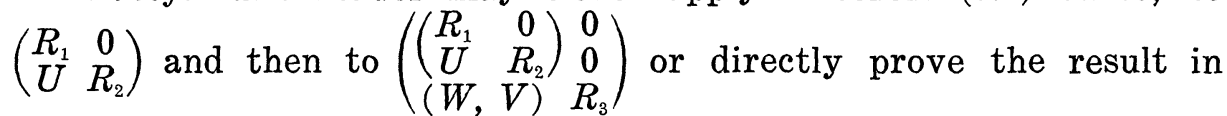
fashion analogous to Theorem (1.5).

The remainder of this paper is devoted to showing that under suitable hypothesis, the study of $\operatorname{Mod}(\Gamma)$ where $\Gamma$ is of form (0.3) may be reduced to the study of $\operatorname{Mod}(\Sigma)$ for some "simpler" ring [of the form (1.6)]. We call such results "separation" theorems for obvious reasons.

2. The main separation result. (2.1) Let $\Gamma$ be a ring of form (0.3). For the remainder of the paper we study $\operatorname{Mod}(\Gamma)$ under the assumption that $\varphi: M \otimes_{\Lambda_{1}} N \rightarrow \Lambda_{2}$ is the zero map. Note that if $\varphi=0$ then property (1.2) is equivalent to the image of $\psi$ is contained in $\left(\operatorname{ann} M_{A_{1}}\right) \cap\left(\operatorname{ann}_{\Lambda_{1}} N\right)$ where ann $M_{\Lambda_{1}}\left(\right.$ resp. $\left.\operatorname{ann}_{A_{1}} N\right)$ denotes the right (resp. left) annihilator of $M($ resp. $N)$ in $\Lambda_{1}$.

In this section we will be concerned with the case where $\Lambda_{1}$ is a left Artin ring with Jacobson radical $r$ where $r^{2}=0$. Before proceeding we state the following basic result:

(2.2) Theorem. [1, §4]. Let $\Lambda$ be a left Artin ring with Jacobson radical $r$ so that $r^{2}=0$. Let $G r(\Lambda / r, r)$ be the category whose objects are triples $(X, Y, f)$ where $X, Y \in \operatorname{Mod}(\Lambda, r)$ and $f \in \operatorname{Hom}_{A / r}\left(r \bigotimes_{\Lambda / r} X, Y\right)$ $i s$ a surjection. The morphisms of $G r(\Lambda / r / r)$ are pairs of maps $\left(\alpha_{1}, \alpha_{2}\right):(X, Y, f) \rightarrow\left(X^{\prime}, Y^{\prime}, f^{\prime}\right)$ where $\alpha_{1} \in \operatorname{Hom}_{{ }^{\prime r}}\left(X, X^{\prime}\right)$ and $\alpha_{2} \in$ $\operatorname{Hom}_{A / r}\left(Y, Y^{\prime}\right)$ so that the following diagram commutes

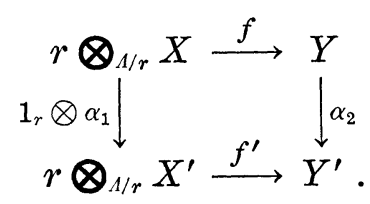


Let $H: \operatorname{Mod}(\Lambda) \rightarrow G r(\Lambda / r, r)$ be the functor defined by $H(A)=$ $\left(A / r A, r A, f_{A}\right)$ where $f_{A}: r \bigotimes_{A / r} A / r A \rightarrow r A$ is induced from the multiplication map $r \otimes_{1} A \rightarrow r A$. Then $H$ is representation equivalence; that is, $H$ is full and dense and $A \in \operatorname{Mod}(\Lambda)$ is indecomposable if and only if $H(A)$ is indecomposable in $\operatorname{Gr}(\Lambda / r, r)$.

For the remainder of this section we assume that

(2.3) (a) $\Lambda_{1}$ is a left Artin ring with Jacobson radical denoted $r$.

(b) $r^{2}=0$

(c) $M_{\Lambda_{1}}$ is a right semisimple $\Lambda_{1}$-module

(d) $N_{\Lambda_{1}}$ is a left semisimple $\Lambda_{1}$-module

(e) the image of $\psi$ is contained in $r$.

Note that under the above conditions (2.1) is satisfied. Let $T$ be the ring

$$
\left(\begin{array}{lll}
\Delta & 0 & 0 \\
M & \Lambda_{2} & 0 \\
r & N & \Delta
\end{array}\right)
$$

where $\Delta=\Lambda_{1} / r$ and $T$ has multiplication given by

$$
\left(\begin{array}{ccc}
d_{1} & 0 & 0 \\
m & \lambda & 0 \\
x & n & d_{2}
\end{array}\right) \cdot\left(\begin{array}{ccc}
d_{1}^{\prime} & 0 & 0 \\
m^{\prime} & \lambda^{\prime} & 0 \\
x^{\prime} & n^{\prime} & d_{2}^{\prime}
\end{array}\right)=\left(\begin{array}{lll}
d_{1} d_{1}^{\prime} & 0 & 0 \\
m d_{1}^{\prime}+\lambda m^{\prime} & \lambda \lambda^{\prime} & 0 \\
x d_{1}^{\prime}+\psi\left(n \otimes m^{\prime}\right)+d_{2} x^{\prime} & n \lambda^{\prime}+d_{2} n^{\prime} & d_{2} d_{2}^{\prime}
\end{array}\right) .
$$

We are now in a position to state and prove the main result of this section.

(2.5) THEOREM. Let $\Gamma$ be a ring of the form (0.3) and suppose $\varphi$ is. the zero map. Suppose further that conditions (2.3) are satisfied. Let $T$ be the ring given by (2.4). There is a canonical additive functor $F: \operatorname{Mod}(\Gamma) \rightarrow \operatorname{Mod}(T)$ which has the following properties:

(1) if $A \in \operatorname{Mod}(\Gamma)$ then $F(A)=0 \Leftrightarrow A=0$.

(2) for all but a finite number of nonisomorphic indecomposable $T$-modules, if $B$ is an indecomposable T-module then there exists a $\Gamma$-module $A$ so that $F(A) \cong B$ as $T$-modules.

(3) if $A, A^{\prime} \in \operatorname{Mod}(\Gamma)$ then $F(A) \cong F\left(A^{\prime}\right)$ as $T$-modules if and only if $A \cong A^{\prime}$ as $\Gamma$-modules.

(4) if $A \in \operatorname{Mod}(\Gamma)$ then $F(A)$ is indecomposable as a T-module if and only if $A$ is indecomposable as a $\Gamma$-module.

Proof. We identify $\operatorname{Mod}(\Gamma)$ with $\mathscr{A}(\Gamma)$ and $\operatorname{Mod}(T)$ with $\mathscr{B}(T)$. Define the functor $F: \operatorname{Mod}(\Gamma) \rightarrow \operatorname{Mod}(T)$ as follows. Let $(X, Y, f, g) \in$ $\operatorname{Mod}(\Gamma)$. Set $X^{\prime}=X / r X$ and $X^{\prime \prime}=$ image of $g$. Let $p: X \rightarrow X^{\prime}$ be 
the canonical surjection. Let $X^{\prime \prime \prime}=p\left(X^{\prime \prime}\right)$ and $X_{1}=X^{\prime} / X^{\prime \prime \prime}$. Let $X_{2}=$ (image of $g$ ) $+r X$, (viewing image of $g$ and $r X$ as submodules of $X$ ). First we note that $X_{1} \oplus X_{2}=0 \Leftrightarrow X=0$. Next we show that $f$ induces a $\Lambda_{2}$-homomorphism $f_{1}: M \otimes_{4} X_{1} \rightarrow Y$. Since $M_{A_{1}}$ is semisimple, $f$ induces a $\Lambda_{2}$-homomorphism $f^{\prime}: M \otimes_{4} X^{\prime} \rightarrow Y$. Now, since $\varphi=0$ it follows by (1.3) that $f\left(M \otimes_{\Lambda_{1}}\right.$ (image of $\left.\left.g\right)\right)=0$. We get the following commutative diagram

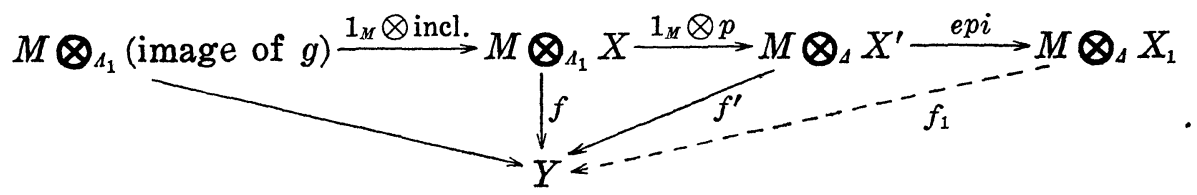

Thus there exists a unique map $f_{1}: M \otimes_{\Delta} X_{1} \rightarrow Y$ making the above diagram commute. Next, it is clear that $g$ induces a map: $g_{1}: N \otimes_{\Lambda_{2}}$ $Y \rightarrow X_{2}$ since (image of $g$ ) $\leqq X_{2}$. Now we have a $\Lambda_{1}$-homomorphism given by multiplication $r \otimes_{\Lambda_{1}} X \rightarrow r X$. This induces a $\Delta$-homomorphism $h^{\prime}: r \bigotimes_{\Delta} X^{\prime} \rightarrow r X$. We claim $h^{\prime}\left(r \otimes_{4} X^{\prime \prime \prime}\right)=0$. We have a split exact sequence

$0 \longrightarrow r X \cap \operatorname{im} g \longrightarrow \operatorname{im} g \longrightarrow X^{\prime \prime \prime} \longrightarrow 0$, where im $g=$ image of $g$.

This sequence is split since im $g \subseteq$ left socle of $X$ which follows from the assumption that $\Lambda_{1} N$ is a semisimple $\Lambda_{1}$-module. Thus the multiplication map $r \otimes_{\Lambda_{1}}$ im $g \rightarrow r X$ is zero. From this it follows that $h^{\prime}\left(r \otimes_{4} X^{\prime \prime \prime}\right)=0$. Since $0 \rightarrow X^{\prime \prime \prime} \rightarrow X^{\prime} \rightarrow X_{1} \rightarrow 0$ is a split exact sequence ( $X^{\prime}$ is a semisimple $\Lambda_{1}$-module), $h^{\prime}\left(r \otimes_{4} X^{\prime \prime \prime}\right)=0$ and $r X \subseteq X_{2}$, we get a canonically induced map $h_{1}: r \otimes_{4} X_{1} \rightarrow X_{2}$. It can be verified, using (1.3) that the following diagram commutes

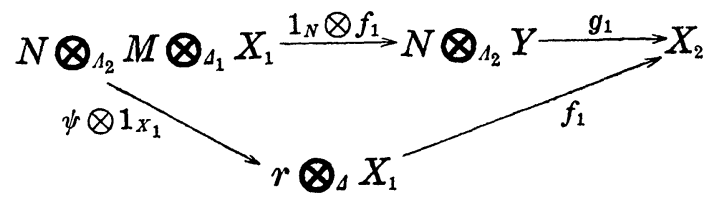

We define $F(X, Y, f, g)=\left(X_{1}, Y, X_{2}, f_{1}, g_{1}, h_{1}\right)$.

We define $F$ on morphisms as follows. If $\left(\alpha_{1}, \alpha_{2}\right):(X, Y, f, g) \rightarrow$ $\left(X^{\prime}, Y^{\prime}, f^{\prime}, g^{\prime}\right)$ is a $\Gamma$-morphism then $\alpha_{1}(\mathrm{im} g) \subseteq \operatorname{im} g^{\prime}$ by (1.4), $\alpha_{1}(r X) \subseteq r X^{\prime}$ and hence $\alpha_{1}\left(x_{2}\right) \subseteq X_{2}^{\prime}$. Thus we get an induced map $\delta_{2}: X_{2} \rightarrow X_{2}^{\prime}$. Since $\alpha_{1}$ induces a map $\bar{\alpha}_{1}: X / r X \rightarrow X^{\prime} / r X^{\prime}$ so that $\bar{\alpha}_{1}$ (in $g / r X \cap \operatorname{im} g$ ) $\subseteq \operatorname{im} g / r X^{\prime} \cap \operatorname{im} g^{\prime}$ we get an induced map $\delta_{1}: X_{1} \rightarrow X_{1}^{\prime}$. We set $F\left(\alpha_{1}, \alpha_{2}\right)=\left(\delta_{1}, \alpha_{2}, \delta_{2}\right)$. Again the reader can verify that $\left(\delta_{1}, \alpha_{2}, \delta_{2}\right)$ is a $T$-morphism.

We now verify properties (1)-(4). Property (1) holds since 
$X_{1} \oplus X_{2}=0 \Leftrightarrow X=0$. We now verify (2). Let $B=(X, Y, Z, f, g, h) \in$ $\operatorname{Mod}(T)$. If $\operatorname{im}(g)+\operatorname{im}(h) \neq Z$, since $\Delta$ is semisimple we have $B \cong$ $\left(X, Y, \quad \operatorname{im} g+\operatorname{im} h, f, g^{\prime}, h^{\prime}\right) \oplus(0,0, Z / \operatorname{im} g+\operatorname{im} h, 0,0,0)$ where $g^{\prime}$ and $h^{\prime}$ are induced from $g$ and $h$. The semisimplicity of $\Delta$ implies there are only a finite number of nonisomorphic indecomposable $T$ modules of the form $(0,0, Z, 0,0,0)$. Hence we may assume $B$ has the property that $Z=\operatorname{im}(g)+\operatorname{im}(h)$. We write $Z=Z_{1} \oplus Z_{2}$ where $Z_{1}=\operatorname{im} h$. We construct $A=(U, V, u, v) \in \operatorname{Mod}(\Gamma)$ as follows. Let $\pi: P \rightarrow X$ be the $\Lambda_{1}$-projective cover of $X$. Then since $r \otimes_{1} X \cong$ $r \bigotimes_{A} P / r P \cong r \bigotimes_{\Lambda_{1}} P \cong r P$ we have the following exact sequence of A-modules:

$$
0 \longrightarrow r \underset{\Delta}{\otimes} X \longrightarrow P \stackrel{\pi}{\longrightarrow} X \longrightarrow 0 \text {. }
$$

Let $U^{\prime}$ be the pushout of

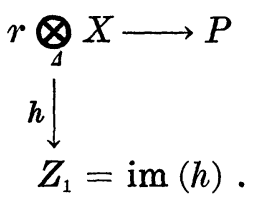

Finally set $U=U^{\prime} \oplus Z_{2}$ and $V=Y$. Note that $r U=r U^{\prime} \cong Z_{1}$ and $U / r U \cong X \oplus Z_{2}$. We define $u: M \otimes_{1} U \rightarrow V$ to be the composition $M \otimes_{\Lambda_{1}} U \stackrel{\rightarrow}{\rightarrow} M \otimes_{4} U / r U \rightarrow M \otimes_{4} X \rightarrow V$. We define $v: N \bigotimes_{\Lambda_{2}} V \rightarrow U$ by $N \otimes_{\Lambda_{2}} V=N \otimes_{\Lambda_{2}} Y \stackrel{g}{\rightarrow} \operatorname{im}(g) \subseteq Z_{1} \oplus Z_{2} \subseteq U^{\prime} \oplus Z_{2}=U$. Using (4.3) one immediately verifies that $A=(U, V, u, v) \in \operatorname{Mod}(\Gamma)$. We now claim $F(A) \cong B$ as $T$-modules. But $\operatorname{im}(v)+r U=Z_{1} \oplus Z_{2}$ and $Z_{1}=r U$. Thus $\operatorname{im}(v) \rightarrow U \rightarrow U / r U$ has image $Z_{2}$ and so $F(A)=\left(X, V, Z_{1} \oplus\right.$ $\left.Z_{2}, u_{1}, v_{1}, u_{2}\right)$. It is a straightforward calculation to show that $F(A) \cong B$ as $T$-modules.

Next we prove property (4). Let $A=(X, Y, f, g) \in \operatorname{Mod}(\Gamma)$. We let $F(A)=\left(X_{1}, Y, X_{2}, f_{1}, g_{1}, h_{1}\right)$ as above. We assme $F(A)$ decomposes:

$$
F(A)=\left(X_{1}^{1}, Y^{1}, X_{2}^{1}, f_{1}^{1}, g_{1}^{1}, h_{1}^{1}\right) \oplus\left(X_{1}^{2}, Y^{2}, X_{2}^{2}, f_{1}^{2}, g_{1}^{2}, h_{1}^{2}\right),
$$

where $X_{i}=X_{i}^{1} \otimes X_{i}^{1}$, for $i=1,2, Y=Y^{1} \oplus Y^{2}, f_{i}=\left(\begin{array}{cc}f_{i}^{1} & 0 \\ 0 & f_{i}^{2}\end{array}\right)$ for $i=1,2$ and $g_{1}=\left(\begin{array}{cc}g_{1}^{1} & 0 \\ 0 & g_{1}^{2}\end{array}\right)$. Set $V_{i}=\operatorname{im} h_{1}^{i}$ for $i=1,2$. Now for $i=1,2$, $g_{1}^{i}: N \otimes_{\Lambda_{2}} Y^{i} \rightarrow X_{2}^{i}$ and $V_{i} \subseteq X_{2}^{i}$. Since im $g_{1}+\operatorname{im} h_{1}=X_{2}$, for $i=1,2$, $\operatorname{im} g_{1}^{i}+\operatorname{im} h_{1}^{i}=X_{2}^{i}$. Let $Z_{i} \subseteq X_{2}^{i}$ so that $X_{2}^{i}=V_{i} \oplus Z_{2}$ and im $g_{1}^{i}=$ (im $\left.g_{1}^{i} \cap V_{i}\right) \oplus\left(\mathrm{im} g_{1}^{i} \cap Z_{i}\right)$. This is possible since $X_{2}^{i}$ is a semisimple $\Delta$-module. Finally, let $V=V_{1} \oplus V_{2}$ and $Z=Z_{1} \oplus Z_{2}$. Then $V=\operatorname{im} h_{1}$. Now $X_{2} \subseteq X$. In particular, $Z \subseteq X$. By choice of $Z$, we have that $Z$ is a semisimple $\Lambda$-module and $Z \nsubseteq \operatorname{im}\left(h_{1}\right)$. But $\operatorname{im}\left(h_{1}\right)=r X$, by construction of $h_{1}$. Thus $X=U \oplus Z$ and we have the following 
easily verified properties:

(a) $r U=\operatorname{im} h_{1}=V$

(b) $U / r U=X_{1}$

(c) the map $h_{1}: r \otimes_{\Delta} X_{1} \rightarrow V$ is induced from the multiplication map $r \otimes_{A_{1}} U \rightarrow r U$. We now apply Theorem (2.2). We see that $H(U)=\left(X_{1}, V, h_{1}\right)$. The decomposition (2.6) yields a decomposition

$$
H(U)=\left(X_{1}^{1}, V_{1}, h_{1}^{1}\right) \oplus\left(X_{1}^{2}, V_{2}, h_{1}^{2}\right) .
$$

By Theorem (2.2), we get a decomposition $U=U_{1} \oplus U_{2}$ so that $H\left(U_{i}\right)=$ $\left(X_{1}^{i}, V_{i}, h_{1}^{i}\right)$ for $i=1,2$. We now leave it to the reader to check that $\left(U_{1} \oplus Z_{1}, Y_{1}, f^{1}, g^{1}\right) \oplus\left(U_{2} \oplus Z_{2}, Y_{2}, f^{2}, g^{2}\right)$ is a decomposition of $(X, Y, f, g)$ where $f^{i}: M \oplus_{i_{1}}\left(U_{i} \oplus Z_{i}\right) \rightarrow Y_{i}$ is defined by the composition

$$
M \bigotimes_{1_{1}}\left(U_{i} \oplus Z_{i}\right) \stackrel{1_{M} \otimes \text { projection }}{\longrightarrow} M \bigotimes_{A_{1}} U_{i} \stackrel{\sim}{\longrightarrow} M \bigotimes_{\Delta} X_{1}^{i} \stackrel{f_{1}^{i}}{\longrightarrow} Y_{i}
$$

and $g^{i}: N \bigotimes_{\Lambda_{2}} Y_{i} \rightarrow U_{i} \oplus Z_{i}$ is defined by the composition

$$
N \bigotimes_{d_{2}} Y_{i} \stackrel{g_{1}^{i}}{\longrightarrow} V_{i} \oplus Z_{i}=r U_{i} \oplus Z_{i} \stackrel{\text { inclusion }}{\longrightarrow} U_{i} \oplus Z_{i} .
$$

Finally, we sketch a proof of property (3). Let $A, A^{\prime} \in \operatorname{Mod}(\Gamma)$ so that $F(A) \cong F\left(A^{\prime}\right)$ as $T$-modules. Let $A=(X, Y, f, g), A^{\prime}=$ $\left(X^{\prime}, Y^{\prime}, f^{\prime}, g^{\prime}\right), F(A)=\left(X_{1}, Y, X_{2}, f_{1}, g_{1}, f_{2}\right)$ and $F\left(A^{\prime}\right)=\left(X_{1}^{\prime}, Y^{\prime}, X_{2}^{\prime}, f_{1}^{\prime}, g_{1}^{\prime}, f_{2}^{\prime}\right)$. Let $\left(\alpha_{1}, \alpha_{2}, \alpha_{3}\right): F(A) \rightarrow F\left(A^{\prime}\right)$ be a $T$-isomorphism. As in the proof of property (4), one may write $X=U \oplus Z$ where $Z$ is a semisimple $\Lambda_{1}$-module, $f\left(M \otimes_{\Lambda_{1}} Z\right)=0$ and $Z \cong$ im g. Similarly $X^{\prime}=U^{\prime} \oplus Z^{\prime}$. Using the functor $H$ : $\operatorname{Mod}\left(\Lambda_{1}\right) \rightarrow G r(\Delta, r)$, as in (4), it is not difficult to show $\left(\alpha_{1}, \alpha_{2}, \alpha_{3}\right)$ induces an isomorphism $H(U) \rightarrow H\left(U^{\prime}\right)$. This lifts to an isomorphism $U \rightarrow U^{\prime}$ by (2.2). Furthermore, by appropriate choice of $Z^{\prime},\left(\alpha_{1}, \alpha_{2}, \alpha_{3}\right)$ induces an isomorphism $Z \rightarrow Z^{\prime}$. Thus we get a $\Lambda_{1}$-isomorphism $\alpha: U \oplus Z \rightarrow U^{\prime} \oplus Z^{\prime}$. Lastly, one verifies that $\left(\alpha, \alpha_{2}\right): A \rightarrow A^{\prime}$ is a $\Gamma$-isomorphism.

We say a ring $R$ is of (left) finite representation type if there are only a finite number of nonisomorphic indecomposable finitely generated left $R$-modules. If $R$ is not of finite representation type we say $R$ is of infinite representation type. As an immediate consequence of Theorem (2.5) we get

(2.7) THEOREM. Let $\Gamma=\left(\begin{array}{ll}\Lambda_{1} & N \\ M & \Lambda_{2}\end{array}\right)$ be such that $\varphi=0$ and (2.3) holds. Then $\Gamma$ is of finite representation type if and only if $T=$ $\left(\begin{array}{lll}\Lambda_{1} / r & 0 & 0 \\ L_{1} & A_{2} & 0\end{array}\right)$ $\left(\begin{array}{lll}M & \Lambda_{2} & 0 \\ r & N & \Lambda_{1} / r\end{array}\right)$ is of finite representation type, where $r=$ Jacobson radical of $\Lambda_{1}$. 
This result has many useful applications. For, assume the hypothesis of (2.5) hold. In general, $\Gamma$ need not have finite left global dimension. But by $[9, \S 4], T$ has finite left global dimension if $\Lambda_{2}$ does. See example (5.3). If, for example, $\Lambda_{2}$ is a semisimple ring then $T$ is a factor ring of a left hereditary ring.

For the remainder of this section, we deal with the case where $\Lambda_{1}$ is semisimple and $\varphi=0$. We show that we automatically get a separation result.

(2.8) Lemma. Under the above hypothesis, the map $\psi: N \otimes_{\Lambda_{2}}$ $M \rightarrow \Lambda_{1}$ is the zero map.

Proof. By (2.1), the image of $\psi=I$ is contained in ann $M_{A_{1}} \cap$ $\operatorname{ann}_{l_{1}} N$. But $I^{2}=I \mathrm{im} \psi$. Thus $I^{2}=I \psi\left(N \otimes_{1_{2}} M\right)=0$. Since $I$ is a two-sided ideal in a semisimple ring we conclude $I=0$.

It now follows that properties (a)-(e) of (2.3) are automatically satisfied. Furthermore, since the Jacobson radical of $\Lambda_{1}$ is $0, T$ is the ring

$$
\left(\begin{array}{ccc}
\Lambda_{1} & 0 & 0 \\
M & \Lambda_{2} & 0 \\
0 & N & \Lambda_{1}
\end{array}\right)
$$

with the obvious multiplication.

Thus, Theorem (2.5) becomes:

(2.9) Theorem. Let $\Gamma$ be the ring $\left(\begin{array}{ll}\Lambda_{1} & N \\ M & \Lambda_{2}\end{array}\right)$ with $\varphi=0$ and $\Lambda_{1}$ a semisimple ring. Let $T$ be the ring $\left(\begin{array}{ccc}\Lambda_{1} & 0 & 0 \\ M & \Lambda_{2} & 0 \\ 0 & N & \Lambda_{1}\end{array}\right)$. There is an additive functor $F: \operatorname{Mod}(\Gamma) \rightarrow \operatorname{Mod}(T)$ so that the following properties hold:

(1) if $A \in \operatorname{Mod}(\Gamma)$ then $F(A)=0 \Leftrightarrow A=0$

(2) for all but a finite number of nonisomorphic indecomposable T-modules, if $B$ is an indecomposable T-module, there is an $\Gamma$ module $A$ so that $B \simeq F(A)$ as T-modules.

(3) if $A, A^{\prime} \in \operatorname{Mod}(\Gamma)$ then $F(A) \cong F\left(A^{\prime}\right)$ as $T$-modules if and only if $A \simeq A^{\prime}$ as $\Gamma$-modules.

(4) if $A \in \operatorname{Mod}(\Gamma)$ then $F(A)$ is an indecomposable $T$-module if and only if $A$ is an indecomposable $\Gamma$-module.

(2.10) COROLLARY. Keeping the hypothesis of (2.9). $\Gamma$ is of finite representation type $\Leftrightarrow T$ is of finite representation type. 
3. The general case with $\varphi=0$. For the remainder of this section we keep the following notations:

$$
\begin{aligned}
\Gamma & =\left(\begin{array}{ll}
\Lambda_{1} & N \\
M & \Lambda_{2}
\end{array}\right) \text { with } \varphi=0 \\
I & =\text { image of } \psi \\
m & =\operatorname{ann} M_{\Lambda_{1}} \\
n & =\operatorname{ann}_{\Lambda_{1}} N .
\end{aligned}
$$

(3.1) Lemma. We have that

(a) $I m=0$

(b) $n I=0$.

Proof. We only prove (a).

$$
I m=\operatorname{Ir}\left(N \otimes_{\Lambda_{2}} M\right) m=0 .
$$

Let

$$
T_{1}=\left(\begin{array}{ccc}
\Delta_{1} & 0 & 0 \\
M & \Lambda_{2} & 0 \\
I & N & \Lambda_{1}
\end{array}\right)
$$

and

$$
T_{2}=\left(\begin{array}{ccc}
\Lambda_{1} / m & 0 & 0 \\
M & \Lambda_{2} & 0 \\
I & N & \Lambda_{1} / n
\end{array}\right)
$$

with the obvious ring structures (see $\S 2$ ). We get

(3.2) Proposition. For $i=1,2$ there are canonical additive functors $G_{i}: \operatorname{Mod}(\Gamma) \rightarrow \operatorname{Mod}\left(T_{i}\right)$.

Proof. We identify $\operatorname{Mod}(\Gamma)$ with $\mathscr{A}(\Gamma)$ and for $i=1,2, \operatorname{Mod}\left(T_{i}\right)$ with $\mathscr{B}\left(T_{i}\right)$. Since the proof is analogous with the first part of Theorem (2.5) we only define the functors on objects and leave the rest to the reader. For $i=1$ define $G_{1}: \operatorname{Mod}(\Gamma) \rightarrow \operatorname{Mod}\left(T_{1}\right)$ as follows: if $A=(X, Y, f, g) \in \operatorname{Mod}(\Gamma)$ let $G_{1}(A)=(X, Y, \operatorname{im}(g), f, g, h)$ where $h$ is induced from the multiplication map $I \otimes_{A_{1}} X \rightarrow X$. For $i=2$, define $G_{2}: \operatorname{Mod}(\Gamma) \rightarrow \operatorname{Mod}\left(T_{2}\right)$ as follows: if $A=(X, Y, f, g) \in \operatorname{Mod}(\Gamma)$ let $G_{2}(A)=(X / m X, Y, \operatorname{im}(g), \bar{f}, g, h)$, where $\bar{f}$ is induced from $f$ and $h$ is induced from the multiplication map $I \otimes_{A_{1}} X \rightarrow X$. Note $n \operatorname{im}(g)=0$ since $n \operatorname{im}(g)=n g\left(N \otimes_{\Lambda_{2}} Y\right)$.

Unfortunately, neither of these functors seem to yield much information in general. But the following result shows that when 
$\psi=0$ one can say something. Note when $\psi=0, T_{1}$ is the ring

$$
\left(\begin{array}{ccc}
\Lambda_{1} & 0 & 0 \\
M & \Lambda_{2} & 0 \\
0 & N & \Lambda_{1}
\end{array}\right)
$$

(3.3) THEOREM. Let $\Gamma=\left(\begin{array}{ll}\Lambda_{1} & N \\ M & \Lambda_{2}\end{array}\right)$ with $\varphi=0$ and $\psi=0$. Let $T_{1}=\left(\begin{array}{ccc}\Lambda_{1} & 0 & 0 \\ M & \Lambda_{2} & 0 \\ 0 & N & \Lambda_{1}\end{array}\right)$. There is a functor $K: \operatorname{Mod}\left(T_{1}\right) \rightarrow \operatorname{Mod}(\Gamma)$ so that if $B \in \operatorname{Mod}\left(T_{1}\right), B$ is a direct summand of $G_{1} K(B)$. In particular, if $\Gamma$ is of finite representation type then so is $T_{1}$.

Proof. We identify $\operatorname{Mod}(\Gamma)$ with $\mathscr{A}(\Gamma)$ and $\operatorname{Mod}\left(T_{1}\right)$ with $\mathscr{B}\left(T_{1}\right)$. Define $K: \operatorname{Mod}\left(T_{1}\right) \rightarrow \operatorname{Mod}(\Gamma)$ as follows: if $B=(X, Y, Z, f, g) \in \operatorname{Mod}\left(T_{1}\right)$ let $K(B)=\left(X \oplus Z, Y, f^{*}, g^{*}\right)$ where $f^{*}: M \otimes_{1}(X \oplus Z) \rightarrow Y \quad$ by $f^{*}(m \otimes(x, z))=f(m \otimes x)$ and $g^{*}: N \otimes, Y \rightarrow X \oplus Z$ by $g^{*}(n \otimes y)=$ $(0, g(n \otimes y))$. Now let $\alpha=\left(\alpha_{1}, \alpha_{2}, \alpha_{3}\right): B \rightarrow G_{1} K(B)$ be defined by $\alpha_{1}: X \stackrel{\left(\begin{array}{l}1 \\ 0\end{array}\right)}{\longrightarrow} X \oplus Z, \alpha_{2}: Y \stackrel{i d}{\longrightarrow} Y$, and $\alpha_{3}: Z \stackrel{\left(\begin{array}{l}0 \\ 1\end{array}\right)}{\longrightarrow} X \oplus Z$. Then $\alpha$ is a $T_{1^{-}}$ monomorphism which is split by $\beta=\left(\beta_{1}, \beta_{2}, \beta_{3}\right): G_{1} K(B) \rightarrow B$ where $\beta_{1}: X \oplus Z \stackrel{(1,0)}{\longrightarrow} X, \beta_{2}: Y \stackrel{i d}{\rightarrow} Y$ and $\beta_{3}: X \oplus Z \stackrel{(1,0)}{\longrightarrow} Z$. Thus $B$ is a direct summand of $G_{1} K(B)$. The last part of the theorem follows immediately.

By example (4.2) we see that $\Gamma$ may be of infinite representation type and yet $T_{1}$ is of finite representation. We conclude this section by giving an application of Theorem (3.3).

(3.4) THEOREM. Let $\Gamma=\left(\begin{array}{ll}A_{1} & N \\ M & \Lambda_{2}\end{array}\right)$ be such that $\varphi=0$. Then if $\Gamma$ is of finite representation type so is the ring

$$
T_{3}=\left(\begin{array}{ccc}
\Lambda_{1} / I & 0 & 0 \\
M & \Lambda_{2} & 0 \\
0 & N & \Lambda_{1} / I
\end{array}\right)
$$

Proof. Since $I \subseteq m \cap n$ by (2.1) $M$ is a right $\Lambda_{1} / I$-module and $N$ is a left $\Lambda_{1} / I$-module. Let $J$ be the two-sided ideal in $\Gamma$ given by $J=\left(\begin{array}{ll}I & 0 \\ 0 & 0\end{array}\right) . \quad$ Note $J^{2}=0$ since $I^{2}=I \psi(N \otimes M)=0$. Let $\bar{\Gamma}=\Gamma / J$. Then if $\Gamma$ is of finite representation type so is $\bar{\Gamma}$. Furthermore, $\bar{\Gamma}=\left(\begin{array}{cc}A_{1} / I & N \\ M & \Lambda_{2}\end{array}\right)$ with $\bar{\varphi}, \bar{\psi}$ the maps induced from $\varphi$ and $\psi$ in $\Gamma$. But $\varphi=0 \Rightarrow \bar{\phi}=0$. Since $I=\operatorname{im}(\psi), \bar{\psi}=0$. The result now follows from Theorem (3.3). 
Example (4.2) shows that the converse is not true.

4. Applications and examples. We begin with an application of Theorem (2.9).

(4.1) Let $\Omega$ be a left Artin ring and let $P$ be a left projective ideal in $\Omega$ such that

(a) $\operatorname{End}_{\Omega}(P)$ is a semisimple ring

(b) if $Q$ and $Q^{\prime}$ are indecomposable projective left $\Omega$-modules and $f: Q \rightarrow Q^{\prime}$ is a nonzero $\Omega$-morphism then $f$ factors through $P \Leftrightarrow$ either $Q$ or $Q^{\prime}$ is isomorphic to a direct summand of $P$.

Then $\Omega$ is of left finite representation type if and only if the ring

$$
\Sigma=\left(\begin{array}{ccc}
\operatorname{End}_{\Omega}(P) & 0 & 0 \\
\operatorname{Hom}_{2}\left(P, P^{*}\right) & \operatorname{End}_{\Omega}\left(P^{*}\right) & 0 \\
0 & \operatorname{Hom}_{\Omega}\left(P^{*}, P\right) & \operatorname{End}_{\Omega}(P)
\end{array}\right)
$$

is of finite representation type where $P \oplus P^{*}$ is a finitely generated projective generator for $\Omega$ such that no direct summand of $P^{*}$ is isomorphic to a direct summand of $P$.

Proof. By the Morita theorems, $\operatorname{Mod}(\Omega)$ is equivalent to $\operatorname{Mod}\left(\operatorname{End}_{\Omega}\left(P \oplus P^{*}\right)^{O P}\right)$ where $R^{o P}$ denotes the opposite ring of $R$.

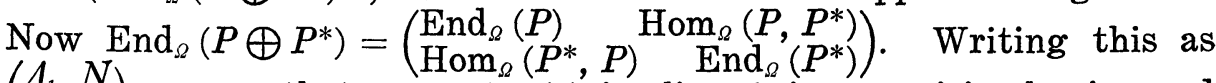
$\left(\begin{array}{ll}A_{1} & N \\ M & \Lambda_{2}\end{array}\right)$ we see that property (a) implies $\Lambda_{1}$ is a semisimple ring and property (b) implies that the multiplication map $M \otimes_{\Lambda_{1}} N \rightarrow \Lambda_{1}$ is zero. Finally noting that a left Artin ring is of finite representation type implies that the ring is right Artin and of finite representation type, we can see the result follows from Theorem (2.9).

(4.2) We now give an example which verifies a number of remarks made in earlier sections.

Let $\Gamma=\left(\begin{array}{ll}\Lambda_{1} & N \\ M & \Lambda_{2}\end{array}\right)$ where $\Lambda_{1}=\Lambda_{2}=k[X] /\left(X^{2}\right), k$ is a field. Let $M=N=k$ where $k$ is the simple $k[X] /\left(X^{2}\right)$-bimodule. We write elements in $k[X] /\left(X^{2}\right)$ as $a+b X$ where $a, b \in k$. We define multiplication in $\Gamma$ by

$$
\left(\begin{array}{cc}
a+b x & e \\
f & c+d x
\end{array}\right) \cdot\left(\begin{array}{cc}
a^{\prime}+b^{\prime} x & e^{\prime} \\
f^{\prime} & c^{\prime}+d^{\prime} x
\end{array}\right)=\left(\begin{array}{cc}
a a^{\prime}+\left(a b^{\prime}+a^{\prime} b\right) x & a e^{\prime}+e c^{\prime} \\
f a^{\prime}+c f^{\prime} & c c^{\prime}+\left(c d^{\prime}+c^{\prime} d\right) X
\end{array}\right) .
$$

Note that the maps $\varphi: M \otimes N \rightarrow \Lambda_{2}$ and $\psi: N \otimes M \rightarrow \Lambda_{1}$ are both zero. Now $\Gamma$ has Jacobson radical $\left(\begin{array}{ll}x k & k \\ k & x k\end{array}\right)$ and its square is zero. Thus applying [5, appendix], since the separated quiver of $\Gamma$ is 


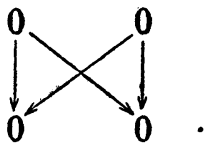

$\Gamma$ is of infinite representation type. We now consider $T_{1}$ of $\S 4$. Namely

$$
T_{1}=\left(\begin{array}{lll}
\Lambda & 0 & 0 \\
M & \Lambda & 0 \\
0 & N & \Lambda
\end{array}\right) .
$$

The ring $T_{1}$ has radical square zero but its separated quiver is<smiles>O=COOO</smiles>

Hence $T_{1}$ is of finite representation type [5, appendix] and we conclude that the converse of Theorem (3.3) does not hold. We now apply the results of $\S 2$. Let $T$ be the ring

$$
\left(\begin{array}{ccc}
k & 0 & 0 \\
k & k[X] / X^{2} & 0 \\
k & k & k
\end{array}\right)
$$

as in $\S 2$. Its separated quiver is

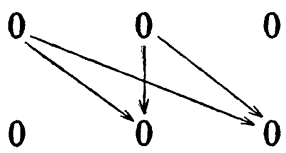

and hence is of infinite representation type (as is also shown by Theorem (2.5)).

(4.3) We conclude with a final example. Let $\Lambda_{1}=\left(\begin{array}{ll}k & k \\ k & k\end{array}\right)$ with $\varphi=0$ and $\psi=0$. Let $\Lambda_{2}=k$. For $i=1,2$ let $S_{i}$ be the simple left $\Lambda_{1}$-module given by $S_{1}=\left(\begin{array}{l}k \\ 0\end{array}\right)$ and $S_{2}=\left(\begin{array}{l}0 \\ k\end{array}\right)$. [Note that by Theorem (2.9), the study of $\operatorname{Mod}\left(\Lambda_{1}\right)$ reduces to the study of $\operatorname{Mod}(T)$ where

$$
T=\left(\begin{array}{lll}
k & 0 & 0 \\
k & k & 0 \\
0 & k & k
\end{array}\right)
$$

with the obvious multiplication.] Let $M=N=k$ where $M_{A_{1}} \cong(0, k)$ 
and ${ }_{\Lambda_{1}} N \cong\left(\begin{array}{l}k \\ 0\end{array}\right):$ Now consider the ring $\Gamma=\left(\begin{array}{ll}\Lambda_{1} & N \\ M & \Lambda_{2}\end{array}\right)$; i.e.,

$$
\left(\begin{array}{lll}
k & k & k \\
k & k & 0 \\
0 & k & k
\end{array}\right)
$$

with multiplication given by

$$
\left(\begin{array}{ccc}
a_{1} & a_{2} & a_{3} \\
b_{1} & b_{2} & 0 \\
0 & c_{2} & c_{3}
\end{array}\right)\left(\begin{array}{ccc}
a_{1}^{\prime} & a_{2}^{\prime} & a_{3}^{\prime} \\
b_{1}^{\prime} & b_{2}^{\prime} & 0 \\
0 & c_{2}^{\prime} & c_{3}^{\prime}
\end{array}\right)=\left(\begin{array}{lll}
a_{1} a_{1}^{\prime} & a_{1} a_{2}^{\prime}+a_{2} b_{2}^{\prime}+a_{3} c_{2}^{\prime} & a_{1} a_{3}^{\prime}+a_{3} c_{3}^{\prime} \\
b_{1} a_{1}^{\prime}+b_{2} b_{1}^{\prime} & b_{1} a_{2}^{\prime}+b_{2} b_{2}^{\prime} & 0 \\
0 & c_{1} b_{2}^{\prime}+c_{3} c_{2}^{\prime} & c_{3} c_{3}^{\prime}
\end{array}\right) .
$$

We note that $\varphi: M \otimes_{1} N \rightarrow \Lambda_{2}$ is the zero map. One easily verifies that $\Lambda_{1}$ has radical square zero and that conditions (2.3) are satisfied. Thus Theorem (2.5) implies that the study of $\operatorname{Mod}(\Gamma)$ can be achieved by studying Mod $(T)$ where $T$ is the ring

$$
\left(\begin{array}{lllll}
k & 0 & 0 & 0 & 0 \\
0 & k & 0 & 0 & 0 \\
0 & k & k & 0 & 0 \\
0 & k & k & k & 0 \\
k & 0 & 0 & 0 & k
\end{array}\right) .
$$

We show

(i) $\Gamma$ has infinite left global dimension

(ii) $\Gamma$ is of radical cubed zero

(iii) $T$ is an hereditary Artin algebra (i.e., gl. dim. $T=1$ )

(iv) $T$ is of finite representation type

(v) $\Gamma$ is of finite representation type.

First we show (i). Consider the simple $\Gamma$-module $\left.\left(\begin{array}{c}k \\ 0 \\ 0\end{array}\right)\right)=S$. (That is, $S \cong S_{1}$ as $\Lambda_{1}$-modules and $\left(\begin{array}{ll}0 & N \\ M & \Lambda_{2}\end{array}\right) S=0$.) Let $0 \rightarrow K \rightarrow$ $P \rightarrow S \rightarrow 0$ be an exact sequence of $\Gamma$-modules with $P \rightarrow S$ the $\Gamma$ projective cover of $S$. Then $\left.K \cong S^{\prime}=\left(\begin{array}{c}0 \\ k \\ 0\end{array}\right)\right)$ where $S^{\prime}$ is the simple $\Gamma$-module which as a $\Lambda$-module is isomorphic to $S_{2}$ and $\left(\begin{array}{ll}0 & N \\ M & \Lambda_{2}\end{array}\right) S^{\prime}=0$. Now let $0 \rightarrow L \rightarrow Q \rightarrow S^{\prime} \rightarrow 0$ be an exact sequence of $\Gamma$-modules so that $Q \rightarrow S^{\prime}$ is the $\Gamma$-projective cover of $S^{\prime}$. Then $S$ is a direct summand of $L$ and we conclude that the left projective dimension of $S$ is infinite. Properties (ii) and (iii) are immediate. Applying [5, appendix], since the quiver of $T$ is

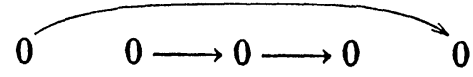


we conclude that $T$ is of finite representation type. Property (v) follows from (2.7). It is worthwhile noting that without Theorems (2.5) and (2.7) there is no known method of attacking the question of whether or not Artin rings of radical cubed zero are of finite representation type.

\section{REFERENCES}

1. M. Auslander, Representation Dimension of Artin Algebras, Queen Mary College Mathematics Notes, London, 1971.

2. M. Auslander, M. I. Platzeck, and I. Reiten, Coxeter functors without diagrams, Trans. Amer. Math. Soc., 250 (1979), 1-46.

3. V. Dlab and C. M. Ringel, Representations of graphs and algebras, Memoirs of the A.M.S. No. 173, 1976.

4. R. M. Fossum, P. A. Griffith, and I. Reiten, Trivial Extensions of Abelian Categories, Lecture Notes in Math., No. 456, Springer-Verlag, Berlin, Heidelberg, New York, 1975. 5. R. Gordon and E. L. Green, Modules with cores and amalgamations of indecomposable modules, Memoir of the A.M.S., No. 187, 1978.

6. E. L. Green, The representation theory of tensor algebras, J. Algebra, 34 (1975), 136-171.

7. E. L. Green and I. Reiner, Integral representations and diagrams, Michigan Math. J., 25 (1978), 53-84.

8. I. Palmer, The global homological dimension of semi-trivial extension of rings, Math. Scand., 37 (1975), 223-256.

9. I. Reiten, Stable equivalence of self-injective algebras, J. Algebra, 40 (1976), 63-74. 10. C. M. Ringel, Representations of k-species and bimodules, J. Algebra, 41 (1976), 269-302.

Received April 11, 1979. This research was partially supported by a grant from the National Science Foudation.

Virginia Polytechnic Institute and State University

BLACKSBURG, VA 24061 


\section{PACIFIC JOURNAL OF MATHEMATICS}

\section{EDITORS}

DONALD BABBITT (Managing Editor)

University of California

Los Angeles, California 90024

Hugo RossI

University of Utah

Salt Lake City, UT 84112

C. C. MOORE and ARTHUR Agus

University of California

Berkeley, CA 94720

\section{J. DUGUNDJI}

Department of Mathematics University of Southern California Los Angeles, California 90007

R. FinN and J. MILGRAM Stanford University Stanford, California 94305

\section{ASSOCIATE EDITORS}
R. ARNES
E. F. BeCKENBACH
B. H. Neumann
F. WOLF
K. YosHIDA

\section{SUPPORTING INSTITUTIONS}

UNIVERSITY OF ARIZONA

UNIVERSITY OF BRITISH COLUMBIA

CALIFORNIA INSTITUTE OF TECHNOLOGY

UNIVERSITY OF CALIFORNIA

MONTANA STATE UNIVERSITY

UNIVERSITY OF NEVADA, RENO

NEW MEXICO STATE UNIVERSITY

OREGON STATE UNIVERSITY
UNIVERSITY OF OREGON UNIVERSITY OF SOUTHERN CALIFORNIA STANFORD UNIVERSITY UNIVERSITY OF HAWAII UNIVERSITY OF TOKYO UNIVERSITY OF UTAH WASHINGTON STATE UNIVERSITY UNIVERSITY OF WASHINGTON 


\section{Pacific Journal of Mathematics}

Vol. 100, No. $1 \quad$ September, 1982

Charalambos D. Aliprantis, Owen Sidney Burkinshaw and M. Duhoux,

Compactness properties of abstract kernel operators $\ldots \ldots \ldots \ldots \ldots \ldots 1$

Roger C. Alperin, Locally compact groups acting on trees .............23

Robert F. Brown, Real homology of Lie group homomorphisms ......... 33

Karen Chase, Maximal groups in sandwich semigroups of binary relations . . 43

W. Wistar (William) Comfort and T. Soundararajan, Pseudocompact

group topologies and totally dense subgroups $\ldots \ldots \ldots \ldots \ldots \ldots \ldots 61$

M. Ferri and C. Gagliardi, Crystallisation moves $\ldots \ldots \ldots \ldots \ldots \ldots \ldots$

Kenneth R. Goodearl, Directly finite aleph-nought-continuous regular

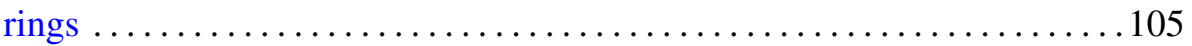

Edward Lewis Green, On the representation theory of rings in matrix

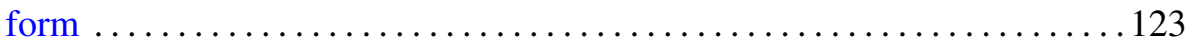

Walter Hengartner and Glenn E. Schober, Interpolation, continuation, and

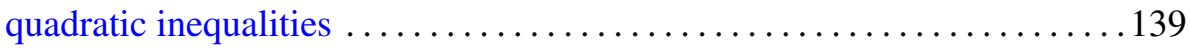

Kenneth Kunen and Haskell Paul Rosenthal, Martingale proofs of some geometrical results in Banach space theory $\ldots \ldots \ldots \ldots \ldots \ldots \ldots \ldots \ldots \ldots$

Brian William McEnnis, Shifts on indefinite inner product spaces. II . . . . . 177

Roman Pol, Note on the spaces $P(S)$ of regular probability measures whose topology is determined by countable subsets $\ldots \ldots \ldots \ldots \ldots \ldots \ldots \ldots 185$

Joan Manuel Verdera Melenchón, Finitely generated projective extensions

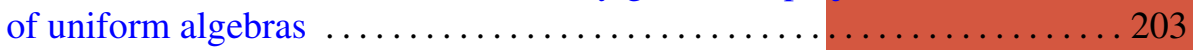

Cheng Ye You, Fixed point classes of a fiber map .................. 217 